\title{
Two-dimensional wetting with binary disorder: a numerical study of the loop statistics
}

\author{
Thomas Garel and Cécile Monthus \\ Service de Physique Théorique, CEA/DSM/SPhT \\ Unité de recherche associée au CNRS \\ 91191 Gif-sur-Yvette cedex, France
}

\begin{abstract}
We numerically study the wetting (adsorption) transition of a polymer chain on a disordered substrate in $1+1$ dimension. Following the Poland-Scheraga model of DNA denaturation, we use a Fixman-Freire scheme for the entropy of loops. This allows us to consider chain lengths of order $N \sim 10^{5}$ to $10^{6}$, with $10^{4}$ disorder realizations. Our study is based on the statistics of loops between two contacts with the substrate, from which we define Binder-like parameters: their crossings for various sizes $N$ allow a precise determination of the critical temperature, and their finite size properties yields a crossover exponent $\phi=1 /(2-\alpha) \simeq 0.5$. We then analyse at criticality the distribution of loop length $l$ in both regimes $l \sim O(N)$ and $1 \ll l \ll N$, as well as the finitesize properties of the contact density and energy. Our conclusion is that the critical exponents for the thermodynamics are the same as those of the pure case, except for strong logarithmic corrections to scaling. The presence of these logarithmic corrections in the thermodynamics is related to a disorder-dependent logarithmic singularity that appears in the critical loop distribution in the rescaled variable $\lambda=l / N$ as $\lambda \rightarrow 1$.
\end{abstract}

\section{INTRODUCTION}

The effect of disorder on the wetting transition in dimension $1+1$ has attracted a lot of interest in the last twenty years and remains a rather controversial issue 1, 2]. The wetting model that we consider here is defined as follows. The (impenetrable) substrate is located at $z=0$. The polymer chain has $N$ monomers, and the position $z_{\alpha}$ of monomer $(\alpha)$ satisfies $z_{\alpha} \geq 0$, with $z_{1}=z_{N}=0$. The partition function of the model reads

$$
Z=\sum_{(R W)} e^{-\beta H}
$$

where $H=\sum_{\alpha=1}^{N} \varepsilon_{\alpha} \delta_{z_{\alpha}, 0}$. In equation (11), the sum runs over all random walks (RW) with $\left|z_{\alpha+1}-z_{\alpha}\right|= \pm 1$ and $\beta=\frac{1}{k_{B} T}$ is the inverse temperature. The contact energies $\left(\varepsilon_{\alpha}\right)$ are independent quenched random variables. We study here the binary distribution $\left(\varepsilon=0\right.$ with probability $p$ and $\varepsilon=\varepsilon_{0}$ with probability $1-p$ ).

On the analytical side, efforts have focused on the small disorder limit [1, 2]. Since the pure wetting transition has a specific heat exponent $\alpha_{\text {pure }}=0$, the disorder is marginal according to the Harris criterion 3]. Based on perturbative calculations, Ref [1] finds a marginally irrelevant disorder (i) the quenched and annealed critical temperatures coincide (ii) the quenched critical properties are the same as in the pure (or annealed) case, up to subdominant logarithmic corrections. Other studies have concluded that that the disorder is marginally relevant 2, 4, 5] (i) the quenched and annealed critical temperatures differ by a term which has an essential singularity in the disorder strength [2] (ii) the critical behavior is governed by some non-trivial disordered fixed point. On the numerical side, the same debate on the disorder relevance took place. Numerical studies for flat and exponential disorder distributions 1], or for binary disorder distribution [6] have concluded that the critical behavior was indistinguishable from the pure transition. On the other hand, the numerical study of [2] for binary disorder pointed towards a negative specific heat exponent $(\alpha<0)$. Finally, the study of Gaussian disorder [7] has been interpreted as an essential singularity in the specific heat, that formally corresponds to an exponent $\alpha=-\infty$. This paper aims at clarifying the situation for the problem defined in equation (11), via the analysis of loop statistics between two contacts with the substrate.

\section{POLAND-SCHERAGA MODEL OF THE WETTING TRANSITION}

\section{A. Model and observables}

The wetting model of eq. (11) is equivalent to the Poland-Scheraga description of DNA denaturation [8, 9]. The relation between the two problems is made apparent if one interprets the coordinate $z$ as the relative coordinate of the two DNA strands. We accordingly define a forward partition function $Z_{f}(\alpha)$ for a chain of $\alpha$ monomers, with 
$z_{1}=z_{\alpha}=0$. From equation (11) we get

$$
Z_{f}(\alpha)=e^{-\beta \varepsilon_{\alpha}} \sum_{\alpha^{\prime}=1}^{\alpha-2} Z_{f}\left(\alpha^{\prime}\right) \mathcal{N}\left(\alpha^{\prime} ; \alpha\right)
$$

where $\mathcal{N}\left(\alpha^{\prime} ; \alpha\right)$ is the the partition function of a loop going from $\alpha^{\prime}$ to $\alpha$. The asymptotic expansion of $\mathcal{N}\left(\alpha^{\prime} ; \alpha\right)$ is given by $[10]$

$$
\mathcal{N}\left(\alpha^{\prime} ; \alpha\right) \simeq \sigma_{0} 2^{\alpha-\alpha^{\prime}} f\left(\alpha-\alpha^{\prime}\right)
$$

where $\sigma_{0}$ is a constant and $f(x)=\frac{1}{x^{c}}$ is the probability to return to the substrate after $\mathrm{x}$ steps. In our model, $c=\frac{3}{2}$. Other values of $c$ are of interest in the DNA denaturation problem [11]. In a similar way, we define a backward partition function $Z_{b}(\alpha)$, defined as the partition function of a chain of $N-\alpha$ monomers, with $z_{\alpha}=z_{N}=0$, which satisfies

$$
Z_{b}(\alpha)=e^{-\beta \varepsilon_{\alpha}} \sum_{\alpha^{\prime}=\alpha+2}^{N} Z_{b}\left(\alpha^{\prime}\right) \mathcal{N}\left(\alpha ; \alpha^{\prime}\right)
$$

In these notations, the partition function $Z$ of equation (11) is given by $Z=Z_{f}(N)=Z_{b}(1)$, and the probability for monomer $\alpha$ to be adsorbed on the substrate is

$$
p(\alpha)=\frac{Z_{f}(\alpha) Z_{b}(\alpha) e^{\beta \varepsilon_{\alpha}}}{Z_{f}(N)}
$$

where the factor $e^{\beta \varepsilon_{\alpha}}$ in the numerator avoids double counting of the contact energy at $\alpha$. The contact density on the substrate (a quantity of primary importance in the DNA context) is given by

$$
\theta_{N}(T)=\frac{1}{N} \sum_{i=1}^{N} p(\alpha)
$$

In the pure case, $\theta_{N}(T)$ is proportional to the energy. Since this is not true in the disordered case, we also consider the contact energy

$$
e_{N}(T)=\frac{1}{N} \sum_{i=1}^{N} \varepsilon_{\alpha} p(\alpha)
$$

We will also be interested in $P_{\text {loop }}(\alpha, \gamma)$, defined as the probability of having a loop between monomers $\alpha$ and $\gamma$ on the substrate

$$
P_{\text {loop }}(\alpha, \gamma)=\frac{Z_{f}(\alpha) \mathcal{N}(\alpha ; \gamma) Z_{b}(\gamma)}{Z_{f}(N)}
$$

\section{B. Numerical implementation}

The above equations, explained in more detail in [12], show that numerical calculations of the partition function $Z$ will require a CPU time of order $O\left(N^{2}\right)$. The Fixman-Freire method [13] reduces this $\mathrm{CPU}$ time to $O(N)$ by approximating the probability factor $f(x)$ of equation (3) by

$$
f(x)=\frac{1}{x^{3 / 2}} \simeq f_{F F}(x)=\sum_{i=1}^{I} a_{i} e^{-b_{i} x}
$$

In equation (9) the number $I$ of couples $\left(a_{i}, b_{i}\right)$ depends on the desired accuracy. The parameters $\left(a_{i}, b_{i}\right)$ are determined by a set of non-linear equations. This procedure has been tested on DNA chains of length up to $N=10^{6}$ base pairs [14, 15], and the choice $I=15$ gives an accuracy better than $0.3 \%$. We have adopted this value throughout this paper.

Putting everything together, the model we have numerically studied is defined by recursion equations (24) for the partition functions where the loop partition function $\mathcal{N}\left(\alpha ; \alpha^{\prime}\right)$ has been replaced by its asymptotic expression (3), with the Fixman-Freire approximation (eq. 9) for $f(x)$. 


\section{LOCALIZATION OF THE CRITICAL TEMPERATURE}

\section{A. Loop statistics and Binder-like parameters}

We now define the probability measure $M_{N}(l)$ for the loops existing in a sample of length $N$ as follows: for each loop length $l$, we sum the loop probability $P_{\text {loop }}(\alpha, \alpha+l)$ (eq.(마) ) over all possible origins $(\alpha)$

$$
M_{N}(l)=\sum_{\alpha=1}^{N-l} P_{\text {loop }}(\alpha, \alpha+l)
$$

The normalization of this measure over $l$ corresponds to the averaged number of loops in a sample of size $N$, or equivalently to the averaged number $N \theta_{N}(T)$ of contacts (6) with the substrate :

$$
M_{N} \equiv \int d l M_{N}(l)=N \theta_{N}
$$

This number is thus extensive $M_{N} \propto N$ in the localized phase $\left(T<T_{c}\right.$ ), and remains finite as $N \rightarrow \infty$ in the delocalized phase $\left(T>T_{c}\right)$.

The first moment of the loop measure $M_{N}(l)$

$$
<l>_{N} \equiv \frac{\int d l l M_{N}(l)}{\int d l M_{N}(l)}
$$

remains finite as $N \rightarrow \infty$ in the localized phase $\left(T<T_{c}\right)$, whereas it diverges as $<l>_{N} \sim N$ in the delocalized phase $\left(T>T_{c}\right)$. We thus introduce the rescaled variable

$$
\lambda=\frac{l}{N}
$$

and the corresponding probability measure $\mathcal{M}_{N}(\lambda)$ for the loops occupying a finite fraction $\lambda=l / N$ of the whole sample. This measure $\mathcal{M}_{N}(\lambda)$ converges respectively in the thermodynamic limit towards $\delta(\lambda)$ in the localized phase $\left(T<T_{c}\right)$ and towards $\delta(\lambda-1)$ in the delocalized phase $\left(T>T_{c}\right)$. At the critical point $T=T_{c}, \mathcal{M}_{N}(\lambda)$ converges for large $N$ towards a stable measure $\mathcal{M}_{c}(\lambda)$ with support $\left.\left.\lambda \in\right] 0,1\right]$, in addition to a singular part in $\delta(\lambda)$ whose weight represents the number of finite loops at criticality.

To locate the critical temperature, it is thus convenient to introduce the following Binder-like parameters 16$]$

$$
B_{N}(T)=\frac{<l^{2}>}{<l>N} \quad, \quad R_{N}(T)=\frac{<l^{3}><l>}{<l^{2}>^{2}}
$$

where $\left\langle l>,\left\langle l^{2}\right\rangle,\left\langle l^{3}>\right.\right.$ are the first moments of the measure $M_{N}(l)$. In the thermodynamic limit $N \rightarrow \infty$, the parameter $B_{\infty}(T)$ jumps from $B_{\infty}\left(T<T_{c}\right)=0$ to $B_{\infty}\left(T>T_{c}\right)=1$. For finite chain lengths $\left(N_{1}, N_{2}, \ldots\right)$, the ratios $B_{N_{1}}(T), B_{N_{2}}(T) .$. cross at $T_{c}$ at some intermediate value $0<B\left(T_{c}\right)<1$ given by

$$
B\left(T_{c}\right)=\frac{<\lambda^{2}>_{c}}{<\lambda>_{c}}
$$

where $<\lambda^{m}>_{c}=\int d \lambda \lambda^{m} \mathcal{M}_{c}(\lambda)$ are the moments of the critical loop measure $\mathcal{M}_{c}(\lambda)$. The parameter $R_{N}(T)$ has a similar behavior with the following crossing value

$$
R\left(T_{c}\right)=\frac{<\lambda>_{c}<\lambda^{3}>_{c}}{<\lambda^{2}>_{c}^{2}}
$$

Computerwise, the evaluation of the full loop distribution $l=1, . ., N$ via eq. (10) requires a time growing as $N^{2}$. To keep a computation time of order $O(N)$, we have chosen to sample the rescaled loop measure $\mathcal{M}_{N}(\lambda)$ on a fixed number $k_{\max }$ of values $\lambda_{k}=k / k_{\max }$ with $k=1,2, . ., k_{\max }$. From this sampling of the loop distribution, we define the reduced moments

$$
<\lambda^{m}>_{k_{\max }}=\sum_{k=1}^{k_{\max }}\left(\frac{k}{k_{\max }}\right)^{m} \mathcal{M}_{N}\left(\lambda_{k}=\frac{k}{k_{\max }}\right)
$$


from which we build the modified Binder parameters

$$
B_{N}^{\left(k_{\max }\right)}(T)=\frac{<\lambda^{2}>_{k_{\max }}}{<\lambda>_{k_{\max }}} \quad, \quad R_{N}^{\left(k_{\max }\right)}(T)=\frac{\left\langle\lambda^{3}>_{k_{\max }}<\lambda>_{k_{\max }}\right.}{<\lambda^{2}>_{k_{\max }}^{2}}
$$

whose properties are the same as the true Binder parameters (14) described above, except for the values of the crossing points that now depend on $k_{\max }$

$$
B^{\left(k_{\max }\right)}\left(T_{c}\right)=\frac{\left\langle\lambda^{2}>_{c, k_{\max }}\right.}{\left\langle\lambda>_{c, k_{\max }}\right.} \quad, \quad R^{\left(k_{\max }\right)}\left(T_{c}\right)=\frac{\left\langle\lambda^{3}>_{c, k_{\max }}<\lambda\right\rangle_{c, k_{\max }}}{<\lambda^{2}>_{c, k_{\max }}^{2}}
$$

where

$$
<\lambda^{m}>_{c, k_{\max }}=\sum_{k=1}^{k_{\max }}\left(\frac{k}{k_{\max }}\right)^{m} \mathcal{M}_{c}\left(\lambda_{k}=\frac{k}{k_{\max }}\right)
$$

We now illustrate these notions on the pure case, before we turn to the analysis of the disordered case.

\section{B. Loop statistics in the pure case}

In the $( \pm 1)$ random walk model (1) with a pure substrate $\left(\varepsilon_{\alpha}=\varepsilon_{0}\right)$, criticality corresponds to the condition $e^{\beta_{c} \varepsilon_{0}}=2$ where the substrate is exactly reflexive [2]. The reflexive nature of the substrate at criticality holds more generally for pure wetting models from a functional RG analysis [17]. This means that the partition function $Z_{N}$ with both ends fixed on the substrate is simply given by the number of random walks returning to the origin after $N$ steps

$$
Z_{N}^{\text {pure }}\left(T_{c}\right) \simeq \frac{2^{N}}{\sqrt{N}}
$$

As a consequence, the critical loop measure (10) reads

$$
M_{N}^{T_{c}}(l) \simeq \frac{\sqrt{N}}{l^{3 / 2}} \int_{1}^{N-l} \frac{d \alpha}{\sqrt{\alpha} \sqrt{N-l-\alpha}} \simeq \frac{\sqrt{N}}{l^{3 / 2}}
$$

i.e. at criticality, there are $\sqrt{N}$ loops, whose lengths are distributed with the random walk first return probability

$$
\rho(l) \sim \frac{1}{l^{3 / 2}}
$$

In terms of the rescaled variable $\lambda=l / N$, the loop measure (23) becomes independent of the size $N$

$$
\mathcal{M}_{c}(\lambda) \simeq \frac{1}{\lambda^{3 / 2}}
$$

This means that at criticality, there are a finite number of loops whose length $l$ represents a finite fraction of the size $N$ of the sample. This property can be understood as follows : the Lévy sum of $n$ independent random variables distributed with (24) scales as $l_{1}+l_{2}+\ldots l_{n} \sim n^{2}$ : as a consequence, the number $n$ of loops scales with the size $N \sim l_{1}+l_{2}+\ldots l_{n}$ of the chain as $n \sim \sqrt{N}$. And for Lévy sums, it is also well known that the maximal length $l_{\text {max }}$ among the $n$ terms of the sum $N \sim l_{1}+l_{2}+\ldots l_{n}$ actually represents a finite fraction of the sum [18], i.e. the biggest loops indeed occupy a finite fraction of the sample.

With the measure (25), the crossing values (1516) are $B\left(T_{c}\right)=1 / 3$ and $R\left(T_{c}\right)=9 / 5$. For our sampling procedure with $k_{\max }$ terms, the crossing values are given in eq.(20), where the moments (21) obtained from (25) read

$$
<\lambda^{m}>_{c, k_{\max }}=\sum_{k=1}^{k_{\max }}\left(\frac{k}{k_{\max }}\right)^{m-3 / 2}
$$

We show in Figure 1 the results of our simulations for the modified Binder parameters (19) for $k_{\max }=100$, $k_{\max }=1000$, and compare them with the calculations of the full Binder parameters (14), which correspond to 

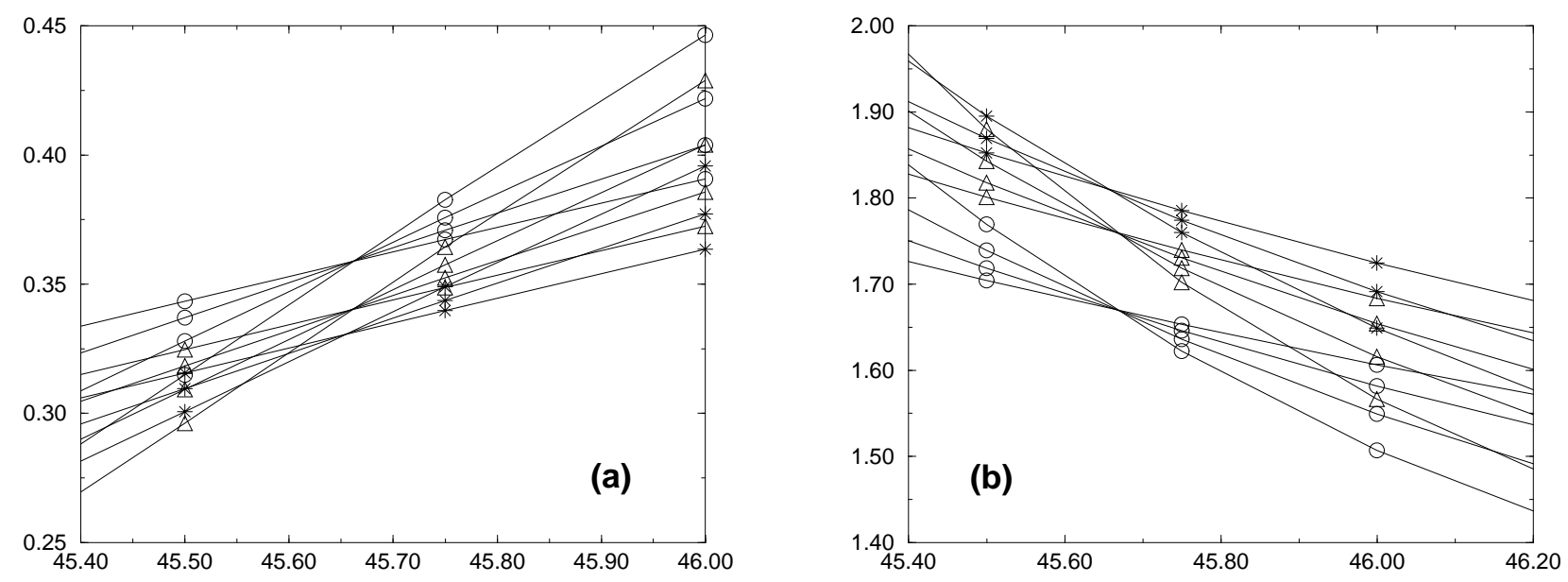

FIG. 1: (a) The Binder parameter $B_{N}^{\left(k_{\max }\right)}(T)$ of the pure case for $k_{\max }=100(\bigcirc), 1000(\triangle), \frac{N}{2}(*)$ and chain lengths up to $N=8 \cdot 10^{5}$. The measured crossing values at the common critical temperature $T_{c}$ are in agreement with eqs (2026) which give 0.3612.. $\left(k_{\max }=100\right)$, 0.3413.. $\left(k_{\max }=1000\right)$, and $\frac{1}{3}\left(k_{\max }=\frac{N}{2}\right)$. (b) The Binder parameter $R_{N}^{\left(k_{\max }\right)}(T)$ of the pure case for $k_{\max }=100(\bigcirc), 1000(\triangle), \frac{N}{2}(*)$ and chain lengths up to $N=8 \cdot 10^{5}$. The measured crossing values at the common critical temperature $T_{c}$ are in agreement with eqs (2026) which give 1.6699..( $\left(k_{\max }=100\right), 1.7584 . .\left(k_{\max }=1000\right)$, and $\frac{9}{5}\left(k_{\max }=\frac{N}{2}\right)$.

$k_{\max }=\frac{N}{2}$. The crossing temperature $T_{c}$ is indeed independent of $k_{\max }$, and the crossing values are in agreement with eqs (20126).

Let us now briefly describe the properties of the loop measure off criticality. The finite-size scaling form of the partition function $Z_{N}$ with both ends fixed on the substrate is [19]

$$
Z_{N}^{\text {pure }}(T) \simeq \frac{2^{N}}{\sqrt{N}} Q\left(\left(T-T_{c}\right) \sqrt{N}\right)
$$

where the function $Q(x)$ satisfies

(i) $Q(x=0)=1$ to recover the critical partition function (22).

(ii) $Q(x \rightarrow-\infty)=-x e^{x^{2}}$ that corresponds to the localized phase

$$
Z_{N}^{\text {pure }}\left(T<T_{c}\right) \underset{N \gg 1 /\left(T-T_{c}\right)^{2}}{\simeq}\left(T_{c}-T\right) 2^{N} e^{\left(T-T_{c}\right)^{2} N}
$$

In this regime, the loop measure (10) reads

$$
M_{N}^{T<T_{c}}(l) \underset{N \gg 1 /\left(\overline{(T}-T_{c}\right)^{2}}{\simeq} N\left(T_{c}-T\right) \frac{e^{-\left(T_{c}-T\right)^{2} l}}{l^{3 / 2}}
$$

i.e. there exists an extensive number $N\left(T_{c}-T\right)$ of finite loops distributed with $\rho_{l o c}(l)=\frac{e^{-\left(T_{c}-T\right)^{2} l}}{l^{3 / 2}}$. The Binder parameters (14) thus converge as $N \rightarrow \infty$ towards $B_{\infty}\left(T<T_{c}\right)=0$ and $R_{\infty}\left(T<T_{c}\right)=3$.

(iii) $Q(x \rightarrow+\infty)=1 / x^{2}$ that corresponds to delocalized phase [2, 17]

$$
Z_{N}^{\text {pure }}\left(T>T_{c}\right) \underset{N \gg 1 /\left(\overline{(T}-T_{c}\right)^{2}}{\sim} \frac{2^{N}}{\left(T-T_{c}\right)^{2} N^{3 / 2}}
$$

The factor $1 / N^{3 / 2}$ means that the substrate becomes repulsive in the delocalized phase [2, 17]. In this regime, the loop measure becomes concentrated on $\delta(\lambda-1)$ and the associated Binder parameters (14) converge towards $B_{N \rightarrow \infty}\left(T>T_{c}\right)=1$ and $R_{N \rightarrow \infty}\left(T>T_{c}\right)=1$.

We show on Figure 2 the loop measures $\mathcal{M}_{N}^{T}(\lambda)$ for two sizes $\left(N=10^{5}\right.$ and $\left.N=4 \cdot 10^{5}\right)$, below, at and above $T_{c}$. At $T_{c}$, the loop measure is independent of $N$ and corresponds to eq (25). Above $T_{c}$, an $N$-dependent minimum shows up. 


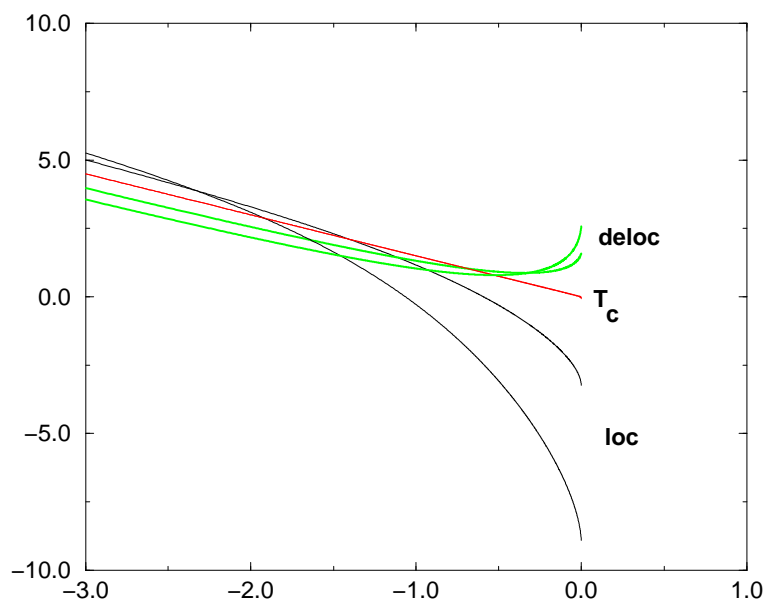

FIG. 2: Log-Log plot of the loop measure $M_{N}^{T}(\lambda)$ of the pure case for $N=10^{5}$ and $N=4 \cdot 10^{5}$, and $T=T_{c}-2\left(\right.$ loc), $T_{c}$ and $T_{c}+2$ (deloc). At $T_{c}$, the two curves are superimposed. Above $T_{c}$, a minimum appears.

\section{Disordered case} $\left(\varepsilon_{\alpha}\right)$

We have numerically studied the wetting transition for the following binary distribution for the contact energies

$$
P\left(\varepsilon_{\alpha}\right)=(1-p) \delta\left(\varepsilon_{\alpha}-\varepsilon_{0}\right)+p \delta\left(\varepsilon_{\alpha}\right)
$$

with the three dilution fractions $p=0.25, p=0.5$ and $p=0.75$.

We have used the sampling method explained above for the loop statistics with the factor $k_{\max }=1000$. We have computed the modified Binder parameters $B_{N}^{\left(k_{\max }\right)}(T)$ and $R_{N}^{\left(k_{\max }\right)}(T)$ (19), for each disorder sample, and for sizes $N=10^{5}, 2 \cdot 10^{5}, 4 \cdot 10^{5}$. Both quantities have been then averaged over $10^{4}$ independent samples (from now on, $\bar{A}$ denotes the disorder average of the quantity $A$ ). The crossing of $\overline{B_{N}^{\left(k_{\max }\right)}}(T)$ and $\overline{R_{N}^{\left(k_{\max }\right)}}(T)$ then yield reasonable error bars in the localization of $T_{c}$ (see Figure 5 ).

\section{Self-averaging properties}

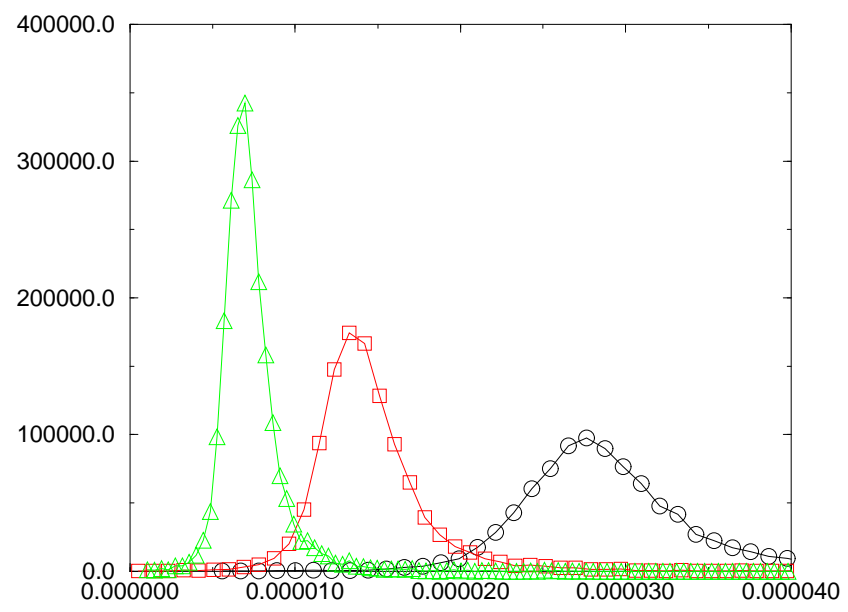

FIG. 3: Histogram over $10^{4}$ disordered samples of $M_{N}\left(l=\frac{N}{2}\right)$ at criticality in the case $p=0.5$, for sizes $N=10^{5}(\bigcirc), 2 \cdot 10^{5}$ $(\square), 4 \cdot 10^{5}(\triangle)$.

In disordered systems, extensive quantities are expected to be self-averaging, because spatial averages in a given sample are equivalent in the thermodynamic limit to disorder averages. For correlation functions, the situation is 
more subtle, as discussed in details in 20. for spin-spin correlations in magnetic systems. Since the loop measure $M_{N}(l)$ (10) is a spatially averaged multiple point correlation function, we have studied its self averaging properties. We show in Figure 3 the histogram over $10^{4}$ disordered samples of $M_{N}\left(l=\frac{N}{2}\right)$ at criticality in the case $p=0.5$, for sizes $N=10^{5}, 2 \cdot 10^{5}, 4 \cdot 10^{5}$ : the distribution of $M_{N}\left(l=\frac{N}{2}\right)$ over the samples is more and more peaked around its average as $N$ grows.

Concerning the crossing of Binder parameters, we have also checked that averaging separately the moments in the numerator and denominator in eqs. (14) gives the same values as the averaged Binder parameters, e.g.

$$
\overline{B_{N}}(T) \simeq \overline{\overline{<l^{2}>}}
$$

This property was also found for usual Binder parameters in magnetic systems [21].

\section{Binder parameters crossings}
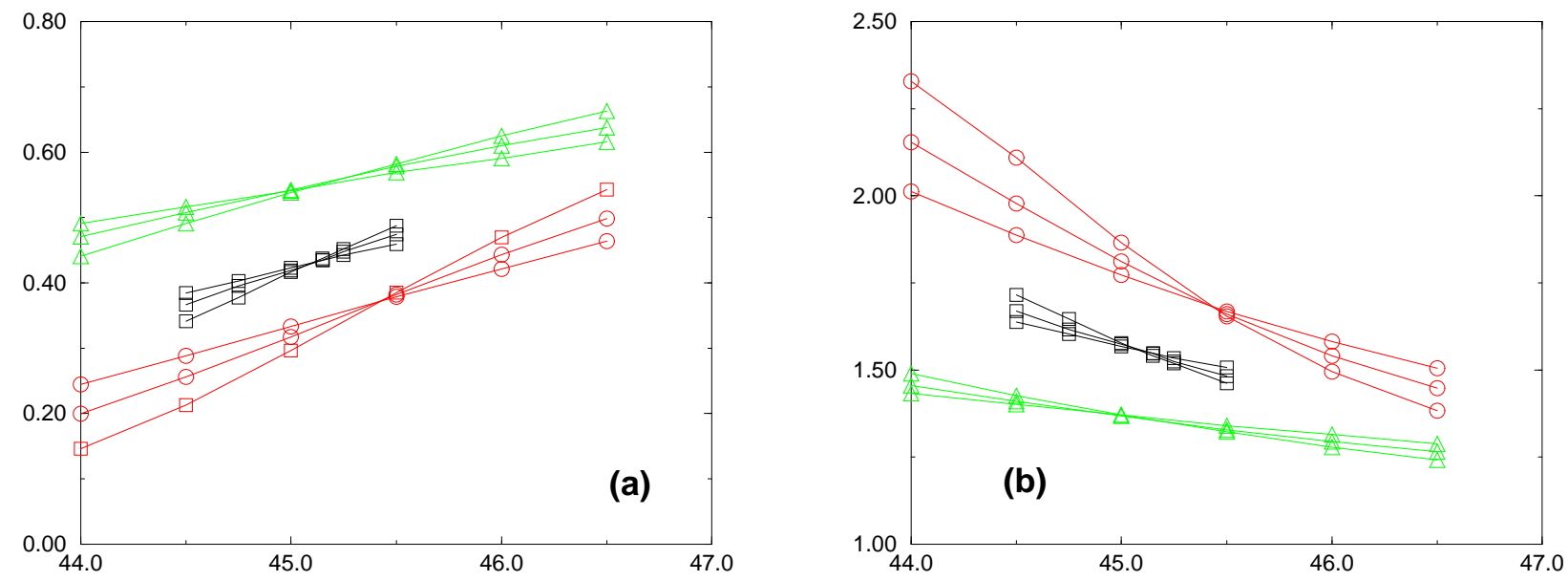

FIG. 4: (a) The averaged Binder parameter $\overline{B^{\left(k_{\max }\right)}} N(T)$, for $k_{\max }=1000,\left(N=10^{5}, 2 \cdot 10^{5}, 4 \cdot 10^{5}\right)$, and $p=$ $0.25(\bigcirc), 0.5(\square), 0.75(\triangle)$. We have rescaled the contact energy $\varepsilon_{0}$ so that the different $T c(p)$ are close. The error bars are much smaller than the symbols. (b) The averaged Binder parameter $\overline{R^{\left(k_{\max }\right)}} N(T)$, for $k_{\max }=1000,\left(N=10^{5}, 2 \cdot 10^{5}, 4 \cdot 10^{5}\right)$, and $p=0.25(\bigcirc), 0.5(\square), 0.75(\triangle)$.
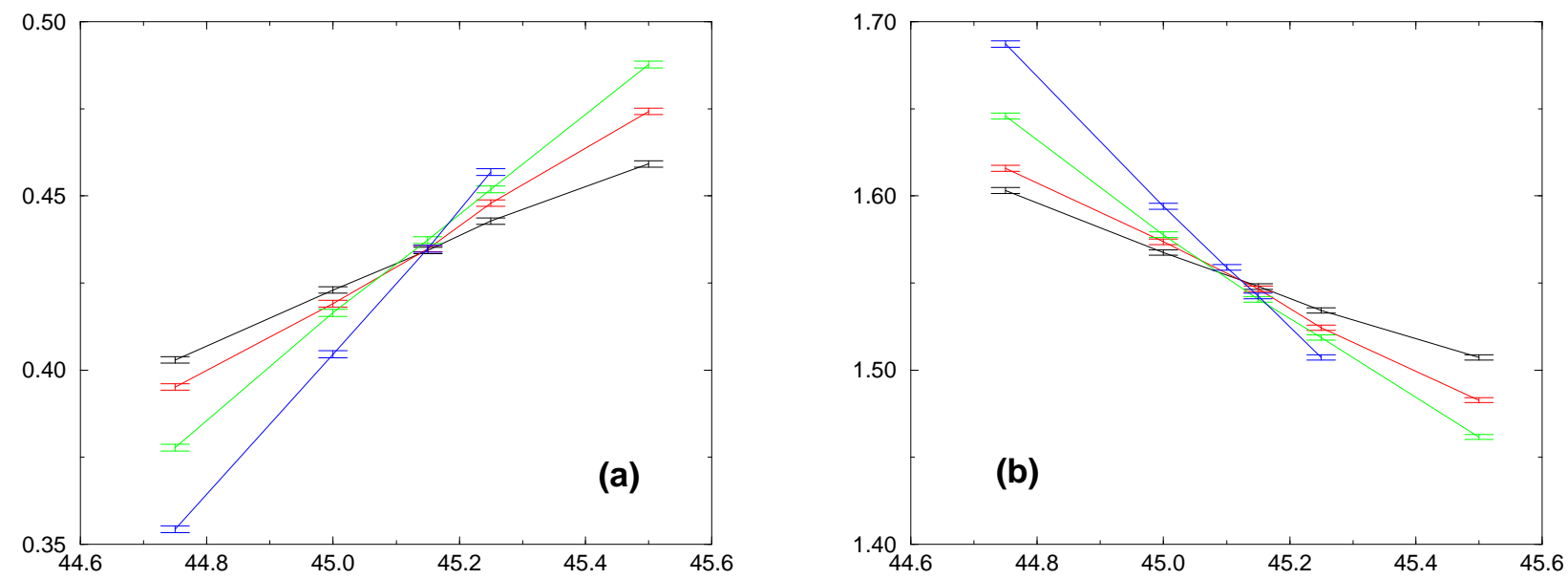

FIG. 5: (a) The averaged Binder parameter $\overline{B_{N}^{\left(k_{\max }\right)}}(T)$, for $k_{\max }=1000$ and $N=1,2,4,8 \cdot 10^{5}$ and $p=0.5$ (b) The averaged Binder parameter $\overline{R_{N}^{\left(k_{\max }\right)}}(T)$, for $k_{\max }=1000$ and $N=1,2,4,8 \cdot 10^{5}$ and $p=0.5$. 
In Figure 4 we show the results for the averaged Binder parameters $\overline{B_{N}^{\left(k_{\max }\right)}}(T)$ and $\overline{R_{N}^{\left(k_{\max }\right)}}(T)$ for sizes $N=$ $1,2,4 \cdot 10^{5}$. The numerical values at the crossing depend on the dilution fraction $p$ (31) :

(i) for $p=0.25$, we obtain $\overline{B^{\left(k_{\max }\right)}}\left(T_{c}\right)=0.370 \pm 0.005$ and $\overline{R^{\left(k_{\max }\right)}}\left(T_{c}\right)=1.685 \pm 0.005$. The critical temperature $T_{c}=45.43 \pm 0.03$ is slightly below the annealed temperature $T_{a n n}=45.50\left(\right.$ here $\left.\varepsilon_{0}=-270\right)$.

(ii) for $p=0.5$, where we have also studied $N=8 \cdot 10^{5}$, we obtain $\overline{B^{\left(k_{\max }\right)}}\left(T_{c}\right)=0.430 \pm 0.005$ and $\overline{R^{\left(k_{\max }\right)}}\left(T_{c}\right)=$ $1.547 \pm 0.005$, with $T_{c}=45.13 \pm 0.03$ as compared to the annealed temperature $T_{a n n}=45.42$. (here $\left.\varepsilon_{0}=-350\right)$.

(iii) for $p=0.75$, we obtain $\overline{B_{N}^{\left(k_{\max }\right)}}\left(T_{c}\right)=0.550 \pm 0.005$ and $\overline{R_{N}^{\left(k_{\max }\right)}}\left(T_{c}\right)=1.360 \pm 0.005$, with $T_{c}=45.15 \pm 0.05$ as compared to the annealed temperature $T_{a n n}=46.82$ (here $\varepsilon_{0}=-515$ ).

These results show that the crossings of Binder parameters allows to locate precisely the critical temperature. We now turn to the analysis of critical properties of various observables.

\section{STUDY OF CRITICAL PROPERTIES}

\section{A. Distribution of loops of length $l \sim O(N)$ at criticality}
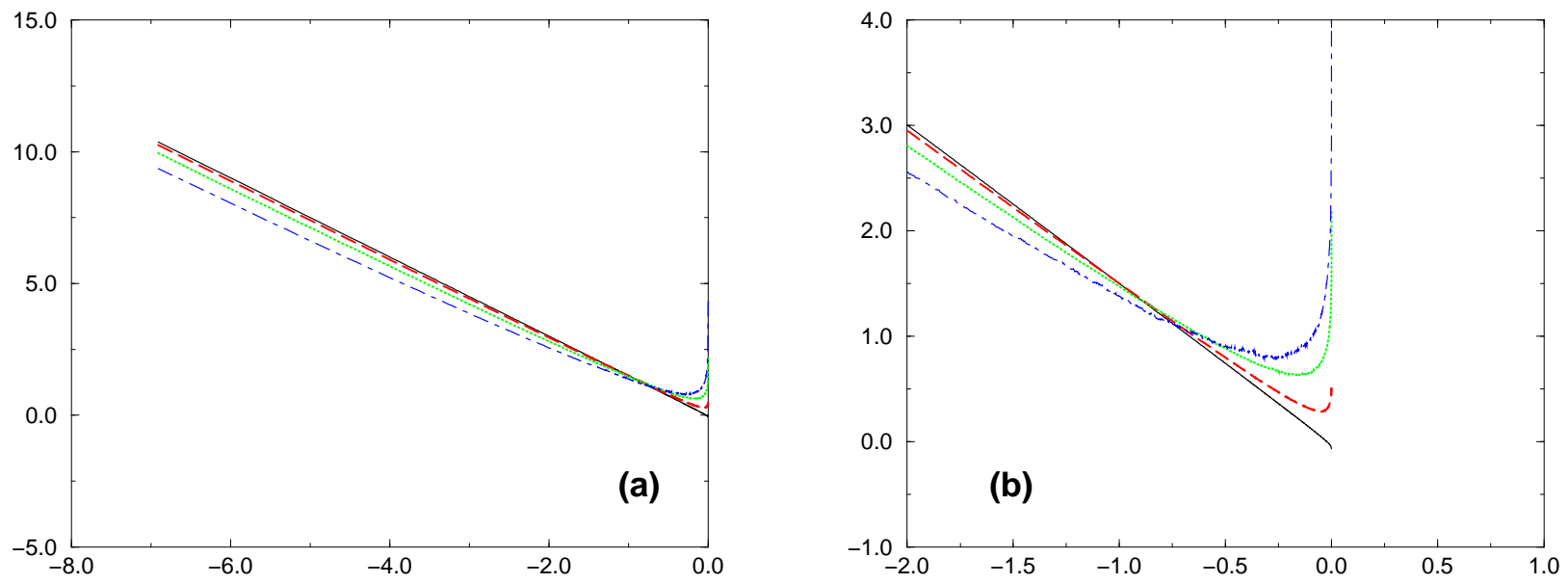

FIG. 6: (a) Log-Log plot of $\overline{\mathcal{M}}_{c}(\lambda)$ for $p=0.25,0.5,0.75$. The pure case $p=0$ (solid black line) is shown for comparison (b) Zoom near $\lambda \rightarrow 1$.

The crossings values $\left(B_{c}, R_{c}\right)$ of the Binder parameters vary continuously with the dilution fraction $p$ (Figure 4 ). This means that the measure $\overline{\mathcal{M}_{c}}(\lambda)$ of loops occupying a finite fraction $\lambda=l / N$ of the sample at criticality also depends continuously on $p$. We present on Figure 6 these loop measures $\overline{\mathcal{M}_{c}}(\lambda)$ for $p=0.25, p=0.5$ and $p=0.75$ in log-log plot, and compare them with the pure case $p=0$, which corresponds to the straight line $\left(\ln \mathcal{M}_{c}(\lambda)=-(3 / 2) \ln \lambda\right.$, eq (25)). In the limit $\lambda \rightarrow 0$ (or $\ln \lambda \rightarrow-\infty$ ), the measures $\overline{\mathcal{M}_{c}}(\lambda)$ become asymptotically parallel to the pure case for all $p$, i.e.

$$
\overline{\mathcal{M}_{c}}(\lambda) \underset{\lambda \rightarrow 0}{\propto} \frac{1}{\lambda^{3 / 2}}
$$

(see also the more detailed study of finite loops below), but otherwise, the loop measures in the disordered cases are qualitatively different from the pure case: a minimum occurs, followed by a weak divergence as $\lambda \rightarrow 1$ (Figure 6 (b)). The form of the divergence suggests that it is logarithmic with a $p$ dependent exponent. The simplest form that can represent the critical loop measure $\overline{\mathcal{M}_{c}}(\lambda)$ on the full interval $0<\lambda<1$ and that is compatible with all our data is

$$
\overline{\mathcal{M}_{c}}(\lambda) \simeq \frac{1}{\lambda^{3 / 2}}\left(1+\frac{C(p)}{(-\ln \lambda)^{\delta(p)}}\right)
$$

The values $\delta(p=0.25) \simeq 0.1, \delta(p=0.5) \simeq 0.25$ and $\delta(p=0.75) \simeq 0.4$ for the exponent $\delta$, and the common value $C(p=0.25) \sim C(p=0.5) \sim C(p=0.75) \sim 6$ for the amplitude $C$ give good fits of (i) the measures $\overline{\mathcal{M}_{c}}(\lambda)$ on the whole range $\lambda \in[0,1]$, with a correct location of the $p$ dependent minimum, and (ii) to the values of the Binder parameters crossings shown in Figures 4 and 5 
Beyond this numerical evidence, it would be of course interesting to have a theoretical explanation for the appearance of this logarithmic singularity in the loop measure near $\lambda \rightarrow 1$ in the disordered case. The only qualitative argument we can think of at this stage is the following : in the pure case, we have seen that a minimum appears in the loop measure in the delocalized phase (see Figure 21). In the disordered case, one may argue that the minimum of the disordered averaged loop measure $\overline{\mathcal{M}_{c}}(\lambda)$ at criticality comes from the fact that at $T_{c}$, among the disordered samples of size $N$, a fraction of these samples tend to be slightly delocalized, with a minimum in their loop measure $\mathcal{M}_{c}(\lambda)$, whereas the other samples tend to be slightly localized (see Figure 17). In other words, if one imagines to associate to each sample $i$ a sample-dependent pseudo critical temperature $T_{c}^{N}(i)$, as it was done in other disordered systems [22], the presence of the minimum at $\lambda_{\text {min }}<1$ reflects the spreading of the pseudo critical temperatures $T_{c}^{N}(i)$ around the thermodynamic critical temperature $T_{c}$. The fact that the exponent $\delta(p)$ grows with $p$ could be interpreted as a consequence of a growing dispersion of the pseudo-critical temperatures $T_{c}^{N}(i)$ with the strength of the disorder.

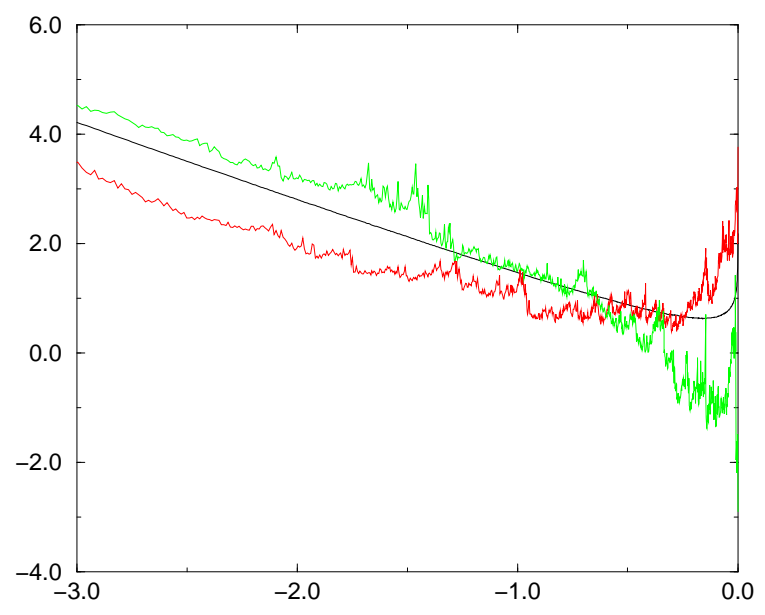

FIG. 7: At criticality for the case $p=0.5$ : Log-Log plot of the averaged loop measure $\overline{\mathcal{M}_{c}}(\lambda)$ over $10^{4}$ samples, as compared to the loop measure of two particular samples. The upward (resp. downward) oriented curve points towards a delocalized (resp. localized) trend

\section{B. Measure of finite loops at criticality}

In the section above, we have discussed the statistics of loops of length $l \sim O(N)$. We now discuss the statistical properties of finite loops, i.e. of length $1 \ll l \ll N$. We have measured the critical loop measure $M_{N}(l)$ for $N=1,2,4 \cdot 10^{5}$ with fixed values of $l(l=10 k ; k=1,2, \ldots, 1000)$. We obtain that the dependence in $l$ is a pure power law

$$
\overline{M_{N}^{T c}}(l) \propto \frac{1}{l^{\gamma(N)}}
$$

with an effective exponent $\gamma(N)$ which increases towards the pure value $3 / 2$ as $N$ increases. For instance for $p=0.5$, we get $\gamma\left(N=10^{5}\right) \simeq 1.46, \gamma\left(N=2 \cdot 10^{5}\right) \simeq 1.468, \gamma\left(N=4 \cdot 10^{5}\right) \simeq 1.475$. This power-law behavior with exponent $3 / 2$ is moreover in agreement with the asymptotic behavior (33) for loops of length $O(N)$ in the limit $\lambda \rightarrow 0$, as it should, since the large $l$ behavior of the finite loop measure $\overline{M_{N}^{T c}}(l)$ should match the small $\lambda \rightarrow 0$ behavior of $O(N)$ loop measure $\overline{\mathcal{M}_{c}}(\lambda)$. This requirement actually determines the $N$-normalization of finite loops. Indeed, we have obtained that at criticality, the measure $\overline{\mathcal{M}_{c}}(\lambda)$ of $O(N)$ loops is independent of the size $N$ and is well described by the form (34). Via the change of variable $l=\lambda N$, this leads to the following normalization for the loop critical distribution (35)

$$
\overline{M_{N}^{T c}}(l) \underset{1 \ll i \ll N}{\simeq} \frac{\sqrt{N}}{l^{3 / 2}}\left(1+\frac{C(p)}{(\ln N)^{\delta(p)}}\right)
$$




\section{Contact density at criticality}

As explained at the beginning, the contact density (6) is directly related to the normalization of the loop measure via (11). The result (36) for the normalization in $N$ of the loop measure thus yields the following finite-size behavior for the contact density at criticality

$$
\overline{\theta_{N}}\left(T_{c}\right) \propto \frac{1}{\sqrt{N}}\left(1+\frac{C(p)}{(\ln N)^{\delta(p)}}\right)
$$

i.e. the leading scaling behavior is the same as in the pure case, but there are strong logarithmic corrections to scaling. Plotting $\left(\sqrt{N} \overline{\theta_{N}}(T)\right)$ for various $N$ thus yields a very poor determination of $T_{c}$, in marked contrast with its precise location through the crossings of the Binder parameters, where these logarithmic corrections are absent.

We have directly computed $\overline{\theta_{N}}\left(T_{c}\right)$ for $N=10^{5}, 2 \cdot 10^{5}$ and $4 \cdot 10^{5}$, for $p=0.25,0.5$ and 0.75 . Our results are in agreement with eq. (37), with the same values of $C(p)$ and $\delta(p)$ quoted above, just after eq. (34). This further supports the form (34) of the critical loop distribution.

\section{Energy at criticality}

We now consider the contact energy (7). In the binary case, it is closely related to the contact density (6), since $\theta_{N}=\frac{e_{N}}{\varepsilon_{0}}+r_{N}$, where $r_{N}$ is the contact density of diluted sites. At criticality, we expect that $r_{N}$ scales at most like $e_{N}$, which implies that the energy $e_{N}\left(T_{c}\right)$ has the same finite size properties as $\theta_{N}\left(T_{c}\right)$, eq. 37.

$$
\overline{e_{N}}\left(T_{c}\right) \propto \frac{1}{\sqrt{N}}\left(1+\frac{C_{e}(p)}{(\ln N)^{\delta(p)}}\right)
$$

with a coefficient $C_{e}(p) \leq C(p)$. The direct measure of the ratios $\frac{e_{N}\left(T_{c}\right)}{\theta_{N}\left(T_{c}\right)}$ increases very slowly with $N$, and typical values for $N=2.10^{5}$ are $0.87(p=0.25), 0.74(p=0.5)$ and $0.6(p=0.75)$.

\section{E. Finite-size scaling in the critical region}
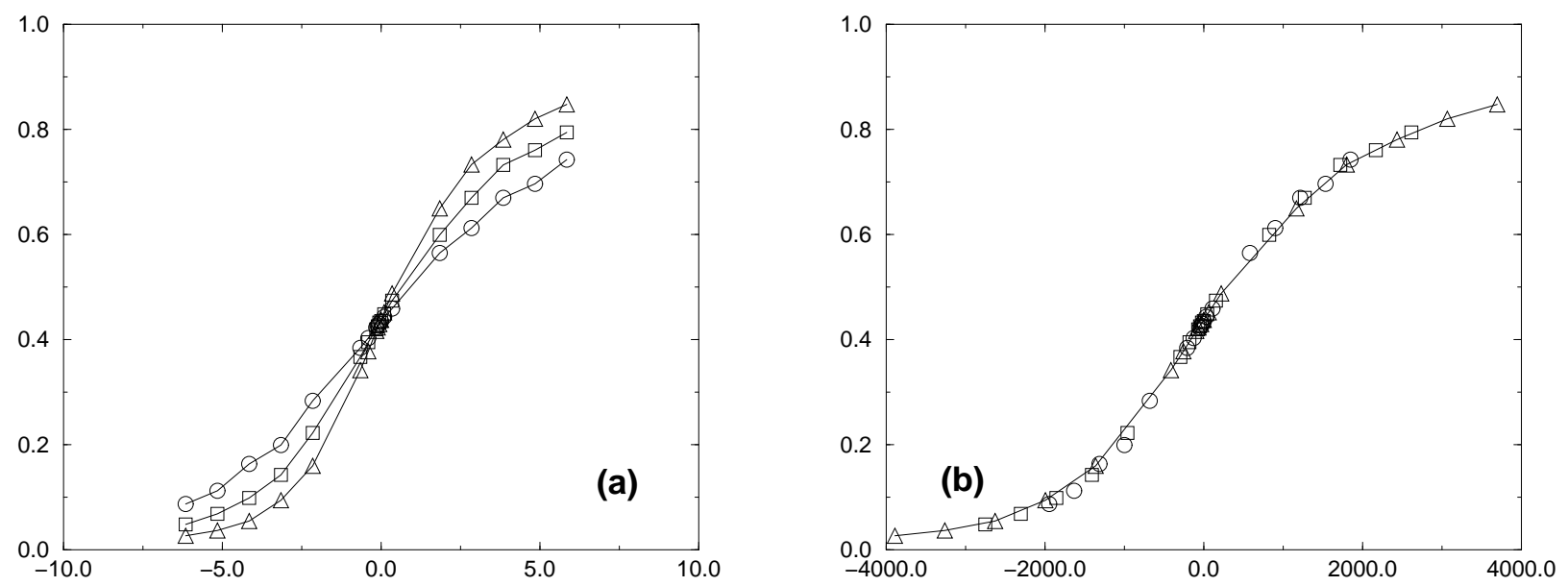

FIG. 8: (a) The Binder parameter $\overline{B_{N}}\left(T-T_{c}\right)$ of the $p=0.5$ case, for $N=10^{5}(\bigcirc), 2 \cdot 10^{5}(\square), 4 \cdot 10^{5}(\triangle)$ (b) Master curve $\mathcal{B}\left(x=\left(T-T_{c}\right) \sqrt{N}\right)$ of equation (39) for the same data and symbols.

We are now interested into the finite-size scaling in the critical region. In terms of the specific heat exponent $\alpha$, the singularity of the free-energy is $f\left(T_{c}\right)-f(T) \sim\left(T_{c}-T\right)^{2-\alpha}$. Via hyperscaling $(f(T) \sim 1 / \xi(T)$ in dimension $d=1$ ), the correlation length along the interface diverges as $\xi(T) \sim 1 /\left(T_{c}-T\right)^{2-\alpha}$. According to finite-size scaling theory, the appropriate rescaled variable is the ratio $N / \xi(T)$ between the size $N$ of the system and this correlation 
length $\xi(T)$. As a consequence, we expect that the Binder parameter $\overline{B_{N}(T)}$ obtained for various sizes $N$ actually only depend on the ratio $N / \xi(T) \sim N\left(T_{c}-T\right)^{2-\alpha}$ or equivalently

$$
\overline{B_{N}}(T)=\mathcal{B}\left(x=\left(T-T_{c}\right) N^{\phi}\right) \text { with } \phi=\frac{1}{2-\alpha}
$$

We show on Figure 8 the master curve obtained with crossover exponent $\phi=1 / 2$, corresponding to $\alpha=0$.

Considering now the finite size scaling for the energy, equations (38) for the energy at $T_{c}$ and the crossover exponent found in (39) suggest the following form

$$
e_{N}(T)=\frac{1}{\sqrt{N}}\left[G_{0}\left(\left(T_{c}-T\right) \sqrt{N}\right)+\frac{1}{(\ln N)^{\delta(p)}} G_{1}\left(\left(T_{c}-T\right) \sqrt{N}\right)+\ldots . .\right]
$$

where the ... represent higher order terms. Our conclusion is that the critical exponents of the binary disordered case are the same as those of the pure case, except for strong corrections to scaling that are $p$ dependent.

\section{CONCLUSIONS AND PERSPECTIVES}

We have studied the two dimensional wetting transition, in the presence of binary disorder for various dilution fraction $p$. Our analysis is based on the probability measure for the loops of length $l$ existing in a sample of size $N$, with both ends of the chain fixed on the substrate. We have first shown how the introduction of Binder-like parameters, built out of the first moments of the loop measure, allows to locate precisely the critical temperature. We have then found numerical evidence that the critical loop distribution $\overline{\mathcal{M}_{c}}(\lambda)$ in the rescaled variable $\lambda=l / N \in[0,1]$ is not a pure power law (in contrast with the pure case), but contains a logarithmic divergence near $\lambda \rightarrow 1$, with a $p$ dependent exponent $\delta(p)$. Finally, we have explained how this singularity in the loop measure induces very strong logarithmic corrections to scaling for the contact density, for the energy, and more generally for thermodynamic quantities.

Our present analysis of the binary disordered case raises the question of the dependence of the critical behavior on the disorder distribution. Indeed, we have obtained that the critical loop distribution $\overline{\mathcal{M}_{c}}(\lambda)$ varies continuously with the dilution fraction $p$ of the binary distribution. More generally, we might expect that $\overline{\mathcal{M}_{c}}(\lambda)$ could depend upon the disorder distribution itself. If this is the case, do the critical exponents differ from those of the binary case? Since the example of Gaussian disorder [7] has been interpreted in terms of essential singularities of the Kosterlitz-Thouless type, we intend to study other types of disorder distributions to clarify this issue.

Another interesting direction concerns the effect of disorder when the a priori loop entropy (3) in the PolandScheraga formulation has an exponent $c>2$, in which case the pure transition is first-order [9]. Indeed, in the context of the DNA denaturation transition, the binding transition between two pure self-avoiding chains on a cubic lattice was found to be first order [23]. The theoretical explanation that has been proposed [1], is that the self-avoidance constraint between denaturated loops and the rest of the chain actually induces an exponent $c>2$ for the loop weight (3). The value $c \sim 2.11$ has been since measured in Monte-Carlo simulations 24]. In the future, we hope to apply our method to the disordered Poland-Scheraga model for the case $c>2$, and to compare with the results recently obtained by B. Coluzzi [25] via Monte-Carlo simulations of self avoiding walks.

Acknowledgements : It is a pleasure to thank B. Coluzzi and J. Houdayer for useful discussions, as well as H. Orland for many contributions over the years.

[1] G. Forgacs, J.M. Luck, Th.M. Nieuwenhuizen and H. Orland, Phys. Rev. Lett., 57, 2184 (1986); J. Stat. Phys., 51, 29 (1988).

[2] B. Derrida, V. Hakim and J. Vannimenus, J. Stat. Phys., 66, 1189 (1992).

[3] A.B. Harris, J. Phys. C 7, 1671 (1974).

[4] S.M. Bhattacharjee and S. Mukherjee, Phys. Rev. Lett., 70, 49 (1993); Phys. Rev. E48, 3483 (1993).

[5] H. Kallabis and M. Lässig, Phys. Rev. Lett., 75, 1578 (1995).

[6] D. Cule and T. Hwa, Phys. Rev. Lett., 79, 2375 (1997).

[7] L-h Tang and H. Chaté, Phys. Rev. Lett., 86, 830 (2001).

[8] R.M. Wartell and A.S. Benight, Phys. Repts., 126, 67 (1985).

[9] D. Poland and H.A. Scheraga eds., Theory of Helix-Coil transition in Biopolymers, Academic Press, New York (1970).

[10] P.G. de Gennes, Scaling concepts in polymer physics, Cornell University Press, Ithaca, (1979).

[11] Y. Kafri, D. Mukamel and L. Peliti, Phys. Rev. Lett., 85, 4988 (2000). 
[12] T. Garel and H. Orland, Biopolymers, 75, 453 (2004).

[13] M. Fixman and J.J. Freire, Biopolymers, 16, 2693 (1977)

[14] R.D. Blake, J.W. Bizarro, J.D. Blake, G.R. Day, S.G. Delcourt, J. Knowles, K.A. Marx and J. SantaLucia Jr., Bioinformatics, 15, 370-375 (1999); see also J. Santalucia Jr., Proc. Natl. Acad. Sci. USA, 95, 1460 (1998).

[15] E. Yeramian, Genes, 255, 139, 151 (2000).

[16] K Binder, Z. Phys., B43, 119 (1981).

[17] D. A. Huse, Phys. Rev. Lett. 58, 176 (1987).

[18] B. Derrida, Physica D 107, 186 (1997).

[19] C. Monthus, T. Garel and H. Orland, Eur. Phys. J. B 17 (2000) 121.

[20] B. Derrida and H. Hilhorst, J. Phys. C 14, L539 (1981).

[21] H. Rieger and A.P. Young, Phys. Rev. Lett., 72, 4141 (1994)

[22] S. Wiseman and E. Domany, Phys. Rev. Lett. 81, 22 (1998) ; Phys Rev E 58, 2938 (1998).

[23] M.S. Causo, B. Coluzzi and P. Grassberger Phys. Rev. E 62, 3958 (2000)

[24] E. Carlon, E. Orlandini and A.L. Stella, Phys. Rev. Lett., 88, 198101 (2002).

[25] B. Coluzzi, in preparation 